(1) CrossMark

Cite this: RSC Adv., 2015, 5, 9618

Received 12th December 2014 Accepted 5th January 2015

\section{Hierarchical-like multipod $\gamma$-MnS microcrystals: solvothermal synthesis, characterization and growth mechanism $\uparrow$}

\author{
Kezhen Qi, ${ }^{a}$ Rengaraj Selvaraj, ${ }^{\text {b }}$ Uiseok Jeong, ${ }^{c}$ Salma M. Z. Al-Kindy, ${ }^{b}$ \\ Mika Sillanpää, ${ }^{d}$ Younghun $\mathrm{Kim}^{\mathrm{c}}$ and Cheuk-wai Tai ${ }^{\mathrm{e}}$
}

DOI: $10.1039 / c 4 r a 16038 j$

www.rsc.org/advances

Novel hierarchical multipod $\gamma$-MnS microcrystals have been successfully synthesized by a simple solvothermal method, in which manganese acetate was used as a manganese source and thiosemicarbazide was used as both sulfur source and capping agent.

As one of the most important semiconductor materials, manganese sulfide $(\mathrm{MnS})$ has attracted great attention because of its potential in extensive applications including optoelectronic, magnetic and luminescent fields. ${ }^{1-4} \mathrm{MnS}$ has three crystal forms: the stable rock-salt $(\alpha-\mathrm{MnS})$, metastable zincblende ( $\beta$-MnS) and wurtzite $(\gamma$-MnS). Compared with the stable form, metastable phases of MnS can be expected to exhibit unique properties. However, the phase-controlled synthesis of MnS crystals still faces many difficulties, because metastable phases of $\beta$-MnS and $\gamma$-MnS suffer from thermal instability and easily transform into the stable $\alpha$-MnS at high temperature or high pressure. ${ }^{5}$ Moreover, since the properties of materials depend on not only their phases, but also on their sizes, shapes and surface structures, much effort has been devoted to synthesize MnS nano/microcrystals with different morphologies such as rods, ${ }^{6}$ spheres,${ }^{7}$ cubes,${ }^{8}$ stars, ${ }^{9}$ corals,${ }^{10}$ flowers, ${ }^{11}$ boxes, ${ }^{12}$ and so on. Among these various morphologies, complex three-dimensional (3D) architectures may offer more opportunities to explore their novel properties, comparing with one-dimensional (1D) and two-dimensional (2D) structures. Especially, 3D branched nano/microstrutures (called

${ }^{a}$ College of Chemistry and Life Science, Shenyang Normal University, Shenyang, 110034, China

${ }^{b}$ Department of Chemistry, College of Science, Sultan Qaboos University, Muscat, Sultanate of Oman. E-mail: srengaraj1971@yahoo.com; Tel: +968-2414-2436

'Department of Chemical Engineering, Kwangwoon University, Seoul 139-701, Korea ${ }^{d}$ Laboratory of Green Chemistry, LUT Savo Sustainable Technologies, Lappeenranta University of Technology, Sammonkatu 12, FI-50130 Mikkeli, Finland

${ }^{e}$ Department of Materials and Environmental Chemistry, Arrhenius Laboratory, Stockholm University, S-106 91 Stockholm, Sweden

$\dagger$ Electronic supplementary information (ESI) available: Experimental details, EDS measurement and SEM image of the sample obtained by adding equimolar sulfur power instead of thiosemicarbazide. See DOI: 10.1039/c4ra16038j multipods) have received particular interest due to their large surface areas, multi-angle edges, and sharp corners. ${ }^{13}$ However, the efficient fabrication of MnS hierarchical multipods still remains a great challenge. Herein, we report a one step solvothermal method to prepare multipod-shaped $\gamma$-Mns hierarchical microstructures, in which manganese acetate was used as manganese resource and thiosemicarbazide was used as both sulfur source and capping agent. Furthermore, a possible growth mechanism of MnS multipods was proposed on the basis of the structural and morphological studies.

The detailed synthetic procedures and characterization methods are listed in the ESI. $\dagger$ The X-ray diffraction (XRD) pattern of the as-prepared $\gamma$-MnS tripods is shown in Fig. 1 . The diffraction pattern can be indexed to a hexagonal phase of $\gamma$-MnS (JCPDS no. 40-1289). Compared with the standard reflections, the intensity of the (002) diffraction peak is decreased, but the (100) peak is increased. This changes in the intensities of (002) and (100) diffraction peaks imply that the growth of $\gamma$-Mns tripods along [001] direction is enhanced, which is consistent with that in the reported literature. ${ }^{14}$

The composition of MnS tripods was further confirmed by XPS analysis (Fig. 2). No peak of other element, except C, O, Mn, and S, was detected in the survey XPS spectrum (Fig. 2a). The observed $\mathrm{C}$ peak is due to the carbon supporting film on the

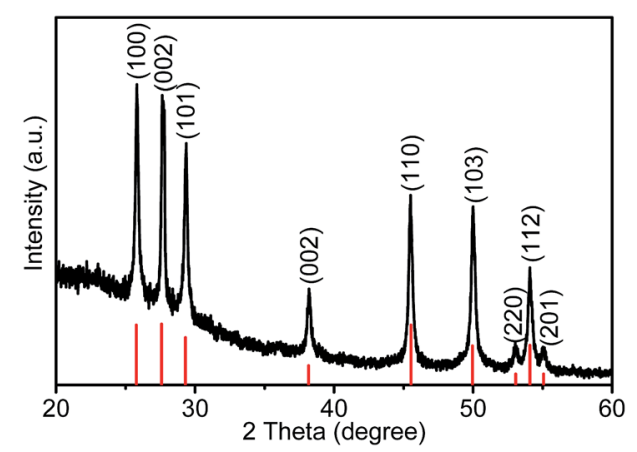

Fig. 1 XRD patterns of the $\gamma$-MnS tripods. 

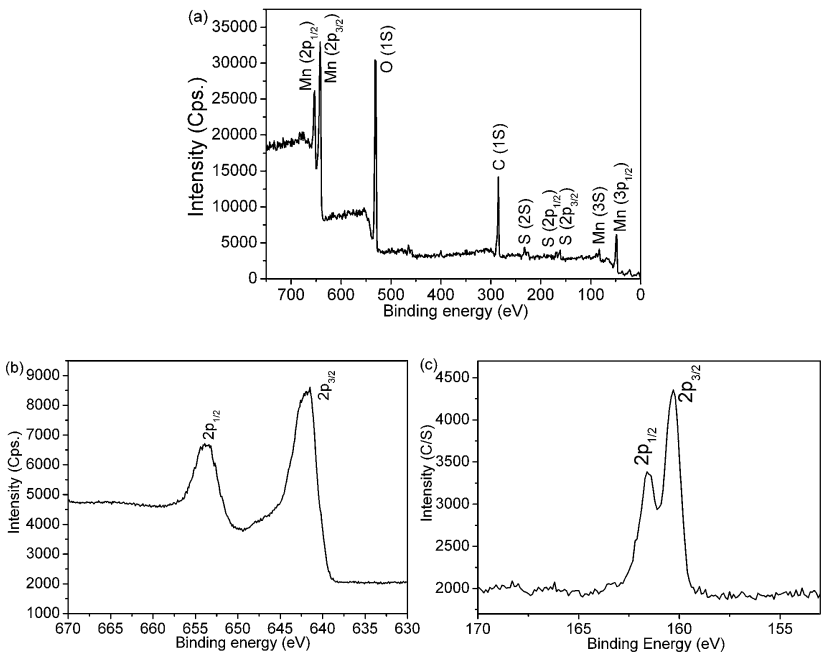

Fig. 2 XPS analysis of the $\gamma$-MnS hierarchical tripods: (a) survey spectrum, (b) Mn $2 p$ and (c) $S 2 p$ binding-energy spectrum.

copper TEM grid, and the $\mathrm{O}$ peak is attributed to the absorption of oxygen on the sample surface or supporting film. The XPS spectra of MnS sample agrees well with the typical MnS spectrum reported in the literature. ${ }^{15} \mathrm{Fig} .2 \mathrm{~b}$ and c showed the highresolution XPS spectra of Mn 3d and S 2p. The peaks at 653.5 and $641.8 \mathrm{eV}$ are assigned to $\mathrm{Mn} 3 \mathrm{~d}_{5 / 2}$ and $\mathrm{Mn} 3 \mathrm{~d}_{3 / 2}$. The peaks at 161.5 and $160.3 \mathrm{eV}$ are attributed to $\mathrm{S} 2 \mathrm{p}_{1 / 2}$ and $\mathrm{S} 2 \mathrm{p}_{3 / 2}$, respectively. Additionally, the energy dispersive X-ray spectroscopy (EDX) analysis also proved that there were no elements other than Mn and S present in the sample (Fig. S1, ESI $\dagger$ ). The EDX analysis indicated that the atomic ratio of $\mathrm{Mn}$ and $\mathrm{S}$ is approximately $1: 1$, which agrees well with the stoichiometry of bulk MnS. Furthermore, the corresponding EDS mapping data provides that $\mathrm{Mn}$ and $\mathrm{S}$ distribute homogeneously throughout the tripods (Fig. S2, ESI†).

Fig. 3a-d shows the typical scanning electron microscopy (SEM) images of as-prepared MnS microcrystals. From the lowmagnification SEM image (Fig. 3a), we can see that the resulting product was present in a large scale of tripods, displaying a special hierarchical microstructure feature. Interestingly, it can be observed that there is an obvious joint in the center, indicating these arms extend from the center. The high-

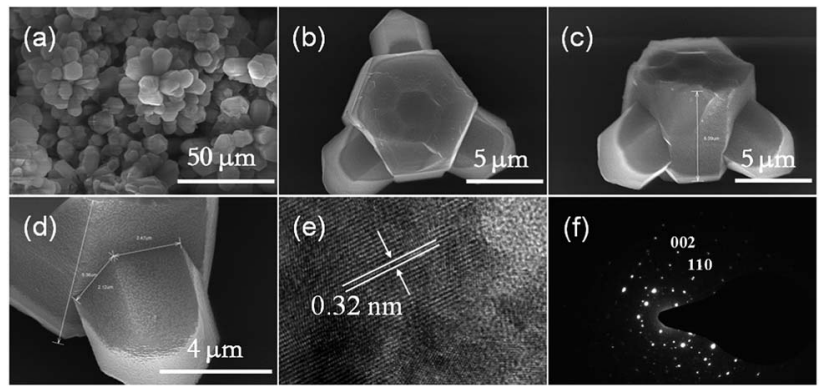

Fig. 3 (a) Low-, and ( $b-d$ ) high-magnification SEM images of asprepared $\gamma$-MnS tripods; (e) HRTEM image located at the top end of the dendrite arm; (f) SAED pattern. magnification SEM images (Fig. 3b-d) exhibited tripod microcrystals with three arthrogenous arms $(\sim 4.0 \mu \mathrm{m}$ in diameter and $\sim 4.5 \mu \mathrm{m}$ in length) which were joined together by a hexagonal column $(\sim 7.5 \mu \mathrm{m}$ in diameter and $\sim 6.0 \mu \mathrm{m}$ in length). The high-resolution transmission electron microscopy (HRTEM) image (Fig. 3e) and the selected area electron diffraction (SAED) pattern (Fig. 3f) taken on the individual dendrite arm shows lattice fringes with interplanar spacing of $0.32 \mathrm{~nm}$, which was indexed to (002) planes of hexagonal $\gamma$-Mns. This result indicates that the dendritic arms preferentially grow along the $c$-axis [001] direction, further confirming the characteristic of the XRD pattern (Fig. 1).

The possible growth mechanism of MnS multipods is illustrated in Fig. 4. $\mathrm{S}^{2-}$ ions can directly react with $\mathrm{Mn}^{2+}$ ions and MnS clusters can be formed quickly, which will be used as the growth units. It is well known that the morphology of the products is highly dependent on their intrinsic crystal structures. ${ }^{16}$ The XRD result shows that the as-prepared MnS has a hexagonal close-packed crystal structure (Fig. 1). Thus, after the initial nucleation, MnS growth habit demonstrates a crystalline hexagonal-shape columns (step 1). During this growing process, the surface of the as-growth crystals could be easily absorbed by the thiosemicarbazide moleculars, which will stabilize and protect the facets of MnS crystals, the bigger hexagonal columns will be formed. To elucidate the exact role of thiosemicarbazide played in affecting MnS shape, the control experiments were carried out. When equimolar sulfur powder was used instead of thiosemicarbazide, the obtained MnS products were irregular particles (Fig. S3, ESI†). This comparison indicates that thiosemicarbazide play not only as sulfur source but also capping agent. Then, secondary nucleation occurred on the lateral sidewalls of the hexagonal columns, followed by subsequent growth of the branched structures that provided high-energy sites for nanocrystal growth (step 2). ${ }^{17}$ This process is driven to minimize the interfacial energy between the new crystals and the primary MnS crystal, which lowers the overall energy of the system after the nucleation burst. Prolonging reaction time to $5 \mathrm{~h}$, as shown in Fig. 3a-d, the morphology of the sample exhibits a tripod-like shape (step 3). As the reaction proceeds to $10 \mathrm{~h}$, more and longer rod-like branches have been formed at the lateral sidewalls of hexagonal columns, finally selfassembling into a hierarchical microstructure (step 4), (Fig. S4, ESI†). This is similar to the formation process of these hierarchical multipods reported previously, such as $\mathrm{ZnO}{ }^{18}$ $\mathrm{CdS},{ }^{19}$ and so on.

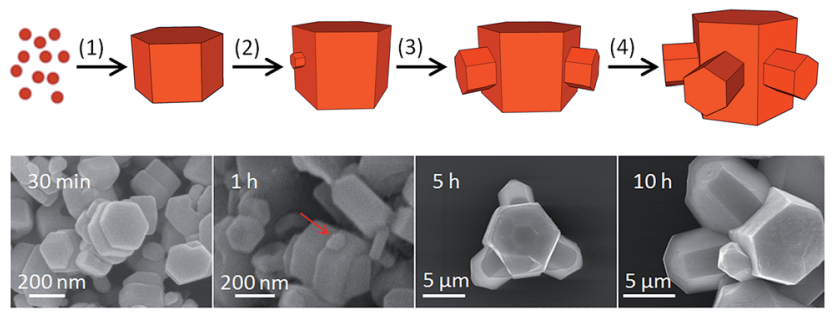

Fig. 4 Schematic illustration of the possible growth mechanism of the $\gamma$-MnS multipods and corresponding SEM images of time dependent. 
In summary, hierarchical multipod $\gamma$-MnS microstructures were successfully synthesized with thiosemicarbazide as both sulphur source and stabilizer. The multipod $\gamma$-MnS microcrystals consisted of three (or six) arthrogenous dendritic arms which were joined together by a hexagonal column. On the basis of the structural and morphological studies, a growth mechanism has been proposed to explain the formation of the MnS multipods.

\section{Acknowledgements}

One of the authors (R.S) thanks Sultan Qaboos University for financial support to carryout this work under a SQU Internal Research Grant (IG/SCI/CHEM/14/02).

\section{Notes and references}

1 L. Levy, D. Ingert, N. Feltin, V. Briois and M. P. Pileni, Langmuir, 2002, 18, 1490.

2 Y. H. Zheng, Y. Cheng, Y. S. Wang, L. H. Zhou, F. Bao and C. Jia, J. Phys. Chem. B, 2006, 110, 8284.

3 A. Puglisi, S. Mondini, S. Cenedese, A. M. Ferretti, N. Santo and A. Ponti, Chem. Mater., 2010, 22, 2804.

4 W. Ma, G. Chen, D. Zhang, K. C. Zhou, G. Z. Qiu and X. H. Liu, J. Phys. Chem. Solids, 2012, 73, 1385.

5 X. Y. Yang, Y. N. Wang, K. Wang, Y. M. Sui, M. G. Zhang, B. Li, Y. M. Ma, B. B. Liu, G. T. Zou and B. Zou, J. Phys. Chem. C, 2012, 116, 3292.

6 C. Zhang, F. Tao, G. Q. Liu, L. Z. Yao and W. L. Cai, Mater. Lett., 2008, 62, 246.
7 Y. Cheng, Y. S. Wang, C. Jia and F. Bao, J. Phys. Chem. B, 2006, 110, 24399.

8 X. Y. Yang, Y. N. Wang, Y. M. Sui, X. L. Huang, T. Cui, C. Z. Wang, B. B. Liu, G. T. Zou and B. Zou, Langmuir, 2012, 28, 17811.

9 Q. W. Tian, M. H. Tang, F. R. Jiang, Y. W. Liu, J. H. Wu, R. J. Zou, Y. G. Sun, Z. G. Chen, R. W. Li and J. Q. Hu, Chem. Commun., 2011, 47, 8100.

10 Y. Liu, Y. Qiao, W. X. Zhang, Z. Li, X. L. Hu, L. X. Yuan and Y. H. Huang, J. Mater. Chem., 2012, 22, 24026.

11 J. G. Yu and H. Tang, J. Phys. Chem. Solids, 2008, 69, 1342.

12 L. Zhang, L. Zhou, H. B. Wu, R. Xu and X. W. (David) Lou, Angew. Chem., 2012, 124, 7379.

13 H. Zhu, G. Li, X. C. Lv, Y. X. Zhao, T. Huang, H. F. Liu and J. L. Lia, RSC Adv., 2014, 4, 6535.

14 J. H. Jiang, R. N. Yu, J. Y. Zhu, R. Yi, G. Z. Qiu, Y. H. He and X. H. Liu, Mater. Chem. Phys., 2009, 115, 502.

15 D. S. Kim, J. Y. Lee, C. W. Na, S. W. Yoon, S. Y. Kim and J. Park, J. Phys. Chem. B, 2006, 110, 18262.

16 W. M. Du, X. F. Qian, X. S. Niu and Q. Gong, Cryst. Growth Des., 2007, 7, 2733.

17 J. A. Nucci, R. R. Keller, D. P. Field and Y. ShachamDiamand, Appl. Phys. Lett., 1997, 70, 1242.

18 T. L. Sounart, J. Liu, J. A. Voigt, M. Huo, E. D. Spoerke and B. McKenzie, J. Am. Chem. Soc., 2007, 129, 15786.

19 W. Guo, J. M. Ma, G. S. Pang, C. Y. Wei and W. J. Zheng, J. Mater. Chem. A, 2014, 2, 1032. 